\title{
Public somnambulism: A general lack of awareness of the consequences of increasing medical negligence litigation
}

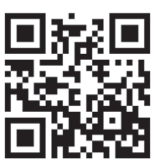

Attend any meeting of obstetricians, spinal surgeons, neurosurgeons or neonatologists, and talk soon turns to the burgeoning costs of cover for negligence claims. Local medical academic and trade journals are increasingly addressing the issue: the whys, the consequences, and possible solutions. ${ }^{[1-4]}$ Although there are regular newspaper headlines and articles on litigation costs, seldom if ever does an article in the lay press address the consequences of increasing medical negligence litigation. Is the public unknowingly sleep-walking into a dystopian future with regard to obstetrics, spinal surgery, neurosurgery and neonatology?

Claims costs depend on the number of claims, the value of those claims, and legal costs. ${ }^{[5]}$ In South Africa (SA), all have increased in recent years. Improved but expensive and sophisticated care has considerably extended life expectancy for extremely compromised patients. Generally speaking, the worse the injury and the longer the survival, the more the costs of care. Those specialties where injuries may be the most severe and survival is likely to be the longest are at greatest risk of extremely high claims; indemnity costs for this group are therefore the highest. It is not surprising, then, that the costs of liability cover for those offering obstetric and neonatal care, spinal surgery and neurosurgery are high and have been increasing rapidly.

Is this a matter that is restricted to obstetricians, spinal surgeons, neurosurgeons or neonatologists in private practice, affecting the income of these specialists, or are there broader issues? While not restricted to these high-risk specialties, there is a tendency for doctors in the current medicolegal environment to practise defensively in an attempt to diminish their medicolegal risk. Defensive medicine is not without its problems, including unnecessary increased costs, the risk attendant to the tests themselves and undue anxiety, to name but a few.

In saving on indemnity costs, some doctors may consider practising without any cover. However, they would be poorly advised to do so, because although an individual may be at a relatively low risk of being claimed against, a single claim, even successfully defended, may ruin them financially. ${ }^{[6]}$ Additionally, there is the question about the ethics of being unindemnified, as deserving patients could be inadequately compensated. Furthermore, it is unlikely that any hospital group would knowingly allow a doctor without adequate indemnity or insurance to practise in a high-risk specialty at one of their hospitals, as claimant lawyers would undoubtedly try to shift the liability onto the hospital group.

The deterioration in claims experience and the impact it has on increasing indemnity costs have impacted on careers across the spectrum of high-risk specialties, and concerns have been raised with regard to recruiting new candidates. ${ }^{[7]}$ Recently qualified specialists may decide that local private practice is unsustainable and remain in state practice (a positive), or leave the country, exacerbating the shortage of doctors, to say nothing of losing the money spent on training them. Established practitioners may either change their practice or retire earlier than anticipated, sometimes directing their attention to medicolegal work and aggravating the situation. A numerically small but extremely important specialty such as neurosurgery is particularly vulnerable to these changes. ${ }^{[8,9]}$

\section{Orthopaedics}

Doctors who operate as a group are more at risk of litigation than those who do not, and orthopaedic surgery is no exception; indeed, they are at relatively high risk even within the context of surgery. Spinal surgery, however, is more expensive to cover as the complications tend to be severe and expensive to compensate. As performing spinal surgery is not universal among orthopaedic surgeons, it would be unfair to expect them all to cover the associated risks and liabilities. An orthopaedic surgeon who performs spinal surgery therefore pays more for cover than one who does not. Initially the difference was relatively modest - a small percentage of the nonspinal rate. Owing to the rapid deterioration in claims experience, the difference has now increased to such an extent that the difference alone is a multiple of the standard orthopaedic rate.

Increasing indemnity costs are already negatively impacting on the orthopaedic spinal surgical community. The differential cost of cover between general orthopaedics, with and without spinal cover, has forced many orthopaedic surgeons to reconsider continuing to perform spinal surgery. This trend is manifested by many resignations of orthopaedic surgeons from the South African Spine Society, citing this very reason. Traditionally, general orthopaedic surgeons would perform the fusion component of spine surgery while operating with a neurosurgical colleague performing the decompressive work. This allowed spinal surgery to function in the smaller cities and towns where there may have been no dedicated spine surgeons. The revenue generated by these procedures often no longer compensates for the additional indemnity cost and risk, resulting in orthopaedic surgeons being unwilling or unable to provide this service. Often the local neurosurgeon is not trained in fusion surgery, with the result that surgery requiring stabilisation is no longer possible outside big cities. Although some may argue that forcing this surgery to the bigger centres and high-volume dedicated spine surgeons is not a bad thing, it disrupts the trauma environment. Spinal trauma frequently presents in smaller towns as a result of high-speed motor vehicle accidents, and there is now paucity of cover. The unindemnified orthopaedic surgeon is reluctant to assess the patient based on the attendant risk; historically neurosurgeons have little to no spine trauma training. This indemnity cost therefore has a direct impact in terms of reducing patient care.

\section{Neurosurgery}

Neurosurgery faces the 'perfect storm' of a numerically small specialty facing high medicolegal risk. ${ }^{[9]}$ While the majority of a private neurosurgeon's operative caseload is adult spinal surgery (which is certainly reflected in their medicolegal risk profile), neurosurgeons also play an important and much broader role in the overall medical community. ${ }^{[9]}$ For example, a private neurosurgeon also treats a wide range of common ailments, many of which are life- or functionthreatening conditions such as traumatic brain injury, stroke or brain tumours. ${ }^{[8,9]}$

Paediatric neurosurgery may be a harbinger of the future. Although society may consider children to be its most precious benisons, this doesn't translate into expenditure on medical care, with paediatric disciplines typically being 'loss leaders' for private hospitals. Remuneration for paediatric surgical procedures often lags far behind that for adult degenerative conditions, to the extent that a private practice limited exclusively to paediatric neurosurgery is unsustainable. Until recently, only three full-time paediatric neurosurgeons in our country offered care to a limited number of highly complex cases from the private sector, but the cost of 


\section{EDITORIAL}

liability cover rendered this unviable. At present, specific paediatric neurosurgery expertise is only available at Red Cross War Memorial Children's Hospital in Cape Town, where the mismatch between operative time and workload now precludes acceptance of patients from the private sector.

\section{Neonatology}

Indemnity costs for paediatricians working with neonates have started to rise. Paediatricians are now being drawn into cerebral palsy claims where had the neonatal care not allegedly been negligent the outcome would have been better. Any neonatal work already increases the cost of cover for a paediatrician by a multiple of the basic paediatric rate. Retinopathy of prematurity claims are high claims, and often involve paediatricians performing neonatal work; ophthalmologists are also increasingly being drawn in. If the cost of covering ophthalmologists who perform neonatal work escalates substantially, ophthalmologists may decline to become involved in the care of these children.

\section{Obstetrics}

Obstetrics is the area where the problem and the consequences are most acute. In the absence of definitive intervention, it is not alarmist to ask who will perform private deliveries by the end of the decade. ${ }^{[10,11]}$ Women will continue to fall pregnant and require delivery, but where will those 100000 -plus deliveries occur? If private obstetricians are unwilling to deliver them, or precluded from doing so, patients will have to deliver in state facilities. Private parturients are unlikely to be enamoured by state hospitals, and the already busy state facilities will be confronted with an increased workload of demanding patients. There is an additional cost burden for the state, as not only will they have to provide the facilities for the extra deliveries, but there will also be a shift of the liability burden for these patients. If and when these patients sue, they will now sue the state. As well as the inconvenience of having to deliver in busier labour wards, state patients will be further disadvantaged, as money allocated to the state's health budget includes provision for litigation. Every rand lost to litigation is a rand lost from state healthcare money set aside for the care of indigent patients.

Significant inflation in the cost of cover for obstetrics has spread further than those performing deliveries. Advances in obstetric ultrasound have enabled identification of fetal anomalies, some of which may lead to severe disabilities. ${ }^{[12]}$ Missing such a case deprives the parents of the opportunity to consider termination of pregnancy. If such a child is delivered and the parents did not have the chance to consider termination, they may sue to cover the costs of the care of the child. Claims for missed abnormalities are emerging as a group that is potentially as expensive a liability as cerebral palsy claims, and this has led to a substantial increase in the cover for obstetric ultrasound. Few non-obstetricians can justify the cost of cover for fetal scanning, thus limiting patients' access to obstetric scanning, and particularly access for those patients living outside urban areas.

\section{What's next?}

So what could the dystopian future entail? Fewer specialists in highrisk specialties, with those remaining practising defensive medicine. An absence, or severe curtailing, of private specialist obstetric care. Paediatricians and ophthalmologists reluctant to manage neonates.
Fewer neurosurgeons in private practice, fewer still with a primary interest in anything other than spinal surgery, and all restricted to the larger urban areas. Likewise few, if any, spinal surgery services outside major urban areas. The problem is not restricted to the private arena, as those patients would now have to be treated in state facilities. Not only are these facilities already busy, but private patients would have to compete for resources and their medicolegal liabilities would move across to the state.

Clearly the issue is far broader than merely affecting the income of doctors in these high-risk specialties. Private patients, private providers, public patients, public providers, politicians and policy pundits all have a vested interest in resolving the problem. The medical profession cannot resolve the issues alone. There is not a medical answer - it has to enter the public debate.

Conflict of interest. GRH is a full-time employee of the Medical Protection Society (MPS). The MPS is not an insurance company but a non-profit mutual organisation.

\section{G R Howarth}

Head of Medical Services - Africa, Medical Protection Society,

2 Victoria House, Victoria Place, Leeds, UK

\section{B Goolab}

President, South African Society of Obstetrics and Gynaecology; Department of Obstetrics and Gynaecology, Faculty of Health Sciences, University of the Witwatersrand, Johannesburg, South Africa

\section{R N Dunn}

Pieter Moll and Nuffield Chair of Orthopaedic Surgery, Department of Orthopaedic Surgery, Faculty of Health Sciences, University of Cape Town, South Africa

\section{A G Fieggen}

Professor and Head, Division of Neurosurgery, Faculty of Health Sciences, University of Cape Town, South Africa

Corresponding author: G R Howarth (graham.howarth@mps.org.uk)

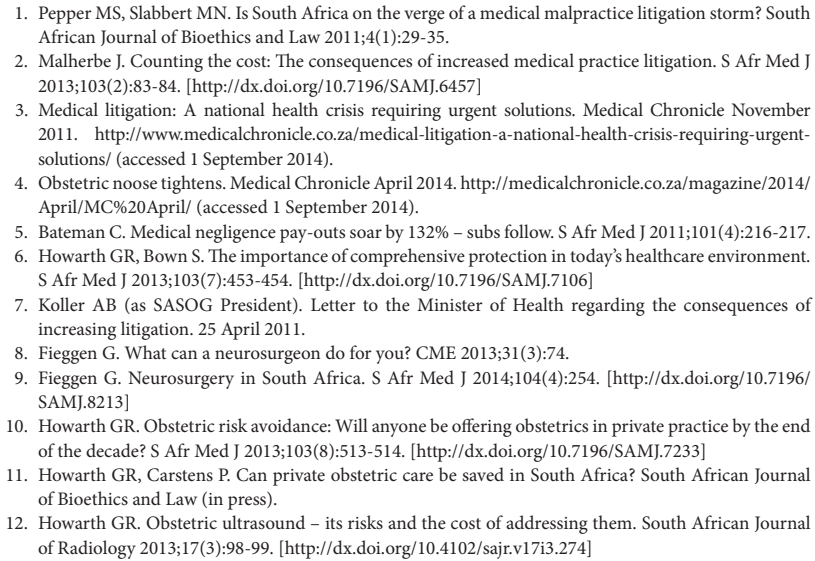

S Afr Med J 2014;104(11):752-753. DOI:10.7196/SAMJ.8568 